\title{
Travelling Wave Solutions of the Perturbed Wadati-Segur-Ablowitz Equation by (G'/G)-Expansion Method
}

\author{
Figen Kangalgil ${ }^{1}$ \\ ${ }^{1}$ Cumhuriyet University, Faculty of Science, \\ Department of Mathematics, 58140, Sivas, Turkey
}

\section{Abstract:}

The investigation of the exact solutions of NLPDEs plays an important role for the understanding of most nonlinear physical phenomena. Also, the exact solutions of this equations aid the numerical solvers to assess the correctness of their results. In this paper, $\left(G^{\prime} / G\right)$-expansion method is presented to construct exact solutions of the Perturbed Wadati-Segur-Ablowitz equation. Obtained the exact solutions are expressed by the hyperbolic, the trigonometric and the rational functions. All calculations have been made with the aid of Maple program. It is shown that the proposed algorithm is elementary, effective and can be used for many PDEs in mathematical physics.

Keywords: The (G'/G) expansion method, Travelling wave solutions, Exact solutions, The Perturbed Wadati-SegurAblowitz equation.

\section{Introduction}

Nonlinear partial differential equations (NLPDEs) are widely used to describe complex phenomena in many fields, such as physics, biology, chemistry, optical fibers, mechanics, etc. So, investigation of exact solutions of NLPDEs will help to understand these phenomena better [1]. Therefore, some researchers have used many powerful methods for obtaining exact solutions of nonlinear partial differential equations, such as inverse scattering method [2], Hirota's bilinear method [3], Backlaund transformation [4], Painleve expansion [5], F-expansion method [24], sine-cosine method [6], homogenous balance method [7, 8], exp-function method $[9,10]$, improved $\left(\mathrm{G}^{\prime} / \mathrm{G}\right)$-expansion method [11, 12, 13, 14, 15], ansatz method [16], the first integral method [17, 18, 19, 20], Kudryashov method, extended trial equation method [21], tanh function method [22, 23], auxiliary equation method [25], differential transform method [26], homotopy perturbation technique [27] and so on.

Recently, the (G'/G)-expansion method was introduced by the Chinese mathematicians Wang et al. [28]. This method is used to find travelling wave solutions of nonlinear partial differential equations. The main idea of this method is that the travelling wave solutions of nonlinear equations can be expressed by polynomial in $\left(\mathrm{G}^{\prime} / \mathrm{G}\right)$, where $G=G(\xi)$ satisfies the second order linear ordinary differential equation $G^{\prime \prime}(\xi)+\lambda G^{\prime}(\xi)+\mu G(\xi)=0 \quad$ where $\xi=x-c t \quad$ and $\lambda, \mu$ and $c$ are constants. The degree of the polynomial can be accomplished by balancing the linear term(s) of highest order with the highest order nonlinear term(s) in the NLPDEs and the coefficients of the polynomial can be obtained by solving a set of algebraic equations resulting from the process of using the proposed method. This method has some pronounced merits over other methods like differential transform method, expfunction method, sine-cosine method, homotopy perturbation method, first integral methods, trial methods, F-expansion method. The solution procedure is direct and simple. The general solution has been obtained this method without approximation. The initial and boundary conditions have not been required. The availability of computer systems like Maple, Mathematica or Matlab facilitates the tedious algebraic calculations. The method is elementary and effective. We have noted that this method changes the given difficult problems which can be solved easily [12]. It will be seen that more travelling wave solutions of many 
NLPDEs. More recently, Zhang et al. [29] applied $\left(G^{\prime} / G\right)$-expansion method to improve Wang et al. s [28] work in order to solve variable coefficient and highdimensional equations. Zhang et al. [30] devised an algorithm for using the method to solve nonlinear differential difference equations. This method is widely used by the references therein $[28,32,33,34,35,36$, $37,38,39,40,41]$.

The aim of this paper is to investigate travelling wave solutions of the Perturbed Wadati-Segur-Ablowitz equation by using the $\left(G^{\prime} / G\right)$-expansion method. The Perturbed Wadati-Segur-Ablowitz equation is as follows: $i u_{x}+u_{t t}+2 \rho\left|u^{2}\right| u-\varepsilon u_{x t}=0$.

Wadati et al. introduced (1.1) to study certain instabilities of the modulated wave trains [42]. "This equation is apparently not integrable because it does not satisfy the Painleve property but it is a Hamiltonian analogue of Kuramoto-Sivashinsky equation which arises in dissipative systems [43]."

\section{The ( $\left.\mathbf{G}^{\prime} / G\right)$-Expansion Method}

In this section, we give main steps of the $\left(G^{\prime} / G\right)$ expansion method $[36,37]$.

Step 1. Take into account a usual NPDE in:

$$
W\left(\mathrm{u}, \mathrm{u}_{t}, \mathrm{u}_{x}, \mathrm{u}_{t t}, \mathrm{u}_{x x}, \mathrm{u}_{x t}, \ldots\right)=0
$$

Then (2.2) transforms the nonlinear ordinary differential equation (NLODE) as

$$
H\left(\mathrm{U}, \mathrm{U}^{\prime}, \mathrm{U}^{\prime \prime}, \mathrm{U}^{\prime \prime}, \ldots\right)=0
$$

such that $u=u(\mathrm{x}, \mathrm{t})=\mathrm{U}(\xi), \xi=\mathrm{x}-\mathrm{ct} \quad$ and $U^{\prime}=\frac{\partial U(\xi)}{\partial \xi} . \quad(2.2)$ is then integrated as long as all terms contain derivatives where integration constants are considered zeros.

Step 2. Suppose that the solutions of (2.2) has a polynomial in $\left(G^{\prime} / G\right)$ as following form:

$U(\xi)=\sum_{i=0}^{m} a_{i}\left(\frac{G^{\prime}}{G}\right)^{i}=\alpha_{m}\left(\frac{G^{\prime}}{G}\right)^{m}+\ldots$

where $a_{i}(i=0,1,2, \ldots, m)$ are constants with $a_{m} \neq 0$ to be determined later.

Step 3. Determine the positive integer $m$ by balancing between the highest order derivatives and highest order nonlinear terms appearing in (2.2). $G=\left(G^{\prime} / G\right)$ satisfies the second order LODE in the form
$G^{\prime \prime}(\xi)+\lambda G^{\prime}(\xi)+\mu G(\xi)=0$

where $\lambda$ and $\mu$ are constants to be determined later.

Step 4. By substituting (2.3) together with (2.4) into (2.2), we get an algebraic equation involving powers of $\left(G^{\prime} / G\right)$. Equating the coefficients of each power of $\left(G^{\prime} / G\right)$ to gives a system of algebraic equations for $a_{i}(i$ $=0,1,2, \ldots, m), \lambda, \mu$ and $c$. By solving this system with aid of Maple or Mathematica, we get unknown constants.

Step 5. The constants $a_{m}, \lambda, \mu c$ and the general solutions of (2.4) into (2.3) we have travelling wave solutions of the (2.1). Solutions of (2.4) depending on whether $\lambda^{2}-4 \mu>0, \lambda^{2}-4 \mu<0, \lambda^{2}-4 \mu=0$,

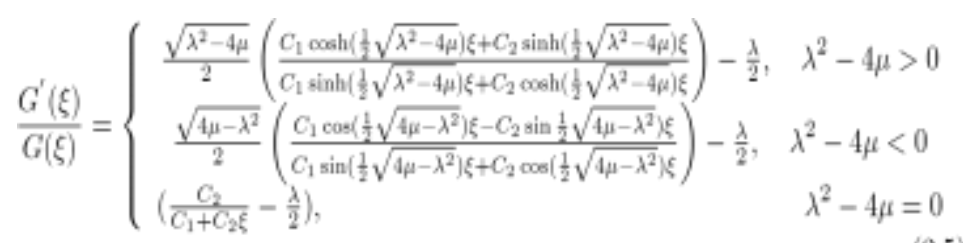

where $C_{1}$ and $C_{2}$ are arbitrary constants.

\section{Applications to the Perturbed Wadati-Segur- Ablowitz Equation}

In this section, we apply the $\left(\frac{r^{\prime}}{f^{\prime}}\right)$-expansion method to construct the travelling wave solutions for the erturbed Wadati-Segur-Ablowitz equation.

\subsection{The Perturbed Wadati-Segur-Ablowitz equation}

Let us consider, the Perturbed Wadati-Segur-Ablowitz equation:

$$
i u_{x}+u_{t t}+2 \rho\left|u^{2}\right| u-\varepsilon u_{x t}=0 .
$$

Let

$$
u(\mathrm{x}, \mathrm{t})=V(\xi) \mathrm{e}^{i \eta}, \xi=x-c t, \eta=c(\mathrm{x}-\sigma \mathrm{t}) .
$$

where $v, c$ and $\sigma$ are constants. After the transform (3.2) and separation of real parts and imaginary parts, we get:

$$
1+2 v c \sigma-c V-c^{2} \sigma^{2} V-\varepsilon c^{2} \sigma V+2 \rho V^{3}=0
$$

and 
$v^{2} V^{\prime \prime}+\varepsilon v V^{\prime}-c V-c^{2} \sigma^{2} V-\varepsilon c^{2} \sigma V+2 \rho V^{3}=0$

Balancing (3.4) we get $m=1$, so the solution of (3.1) is of the form:

$$
V(\xi)=a_{0}+a_{1}\left(\frac{G^{\prime \prime}(\xi)}{G(\xi)}\right), a_{1} \neq 0
$$

Substituting Eqs (2.3) and (2.4) into (3.4), collection the coefficients of $\left(\frac{G^{\prime}}{G}\right)^{m}(\mathrm{~m}=0,1,2,3)$ and set it zero we obtain the system

$\left(\frac{G}{G}\right)^{0}:-c^{2} \sigma^{2} a_{0}+v^{2} \lambda \mu a_{1}+\omega v \lambda \mu a_{1}+2 \rho a_{0}^{3}-c a_{0}-\epsilon c^{2} \sigma a_{0}$

$\left(\frac{G}{G}\right)^{1}: v^{2} \lambda^{2} a_{1}+\epsilon \lambda \lambda^{2} a_{1}-c a_{1}+6 \rho a_{0}^{2} a_{1}-c^{2} \sigma^{2} a 1+2 \epsilon v a_{1} \mu-\epsilon c^{2} \sigma a_{1}+2 v^{2} \mu a_{1}$

$\left(\frac{G}{G}\right)^{2}: 3 \epsilon v \lambda a_{1}+3 v^{2} \lambda a_{1}+6 \rho a_{0} a_{1}^{2}$

$\left(\frac{G^{\prime \prime}}{G}\right)^{3}: 2 e v a_{1}+2 v^{2} a_{1}+2 \rho a_{1}^{3}$

Solving this system (3.6) by MAPLE gives

\section{Case 1:}

$a_{0}= \pm \frac{\sqrt{16} \sqrt{\frac{1}{\rho}} \epsilon \lambda}{16}$,
$a_{1}= \pm \frac{\epsilon \sqrt{16}}{8 \rho \sqrt{\frac{1}{\rho}}}$,
$c=\frac{2}{\epsilon^{2}}, v=-\frac{\epsilon}{2}$
$\sigma=\frac{\left(-2 \pm \frac{\sqrt{-16-8 c^{c} \mu+2 \epsilon^{2} \lambda^{3}}}{2}\right) \epsilon}{4}$,

Case 2: If we take $k_{1}=\frac{\varepsilon v+v^{2}}{4 \rho}$

$a_{1}= \pm \sqrt{-k_{1}} \lambda a_{1}=\mp \frac{2 k_{1}}{\sqrt{-k_{1}}}, r=0$,

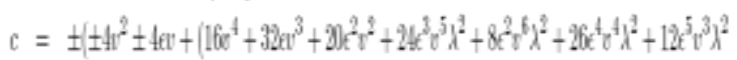

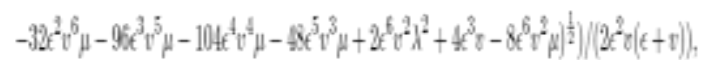

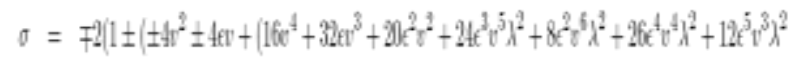

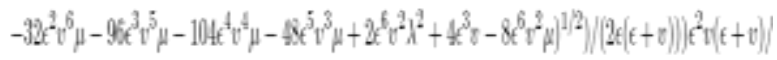

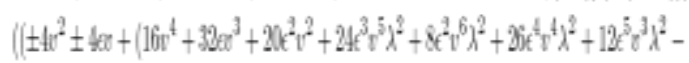

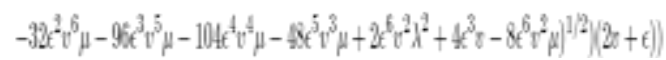

and

$u_{1}(x, t)=\left[ \pm \frac{\epsilon \sqrt{\lambda^{2}-4 \mu}}{4 \sqrt{\rho}} \times\left(\frac{C_{1} \sinh \left(\frac{1}{2} \sqrt{\lambda^{2}-4 \mu}\right) \xi+C_{2} \cosh \left(\frac{1}{2} \sqrt{\lambda^{2}-4 \mu}\right) \xi}{C_{1} \cosh \left(\frac{1}{2} \sqrt{\lambda^{2}-4 \mu}\right) \xi+C_{2} \sinh \left(\frac{1}{2} \sqrt{\lambda^{2}-4 \mu}\right) \xi}\right)\right] \times e^{i \varepsilon(x-\sigma t)}$

where $\xi=x+\frac{\varepsilon}{2} t$.

When $\lambda^{2}-4 \mu<0$, we have the trigonometric type

$$
V_{2}(\xi)= \pm \frac{\epsilon \sqrt{4 \mu-\lambda^{2}}}{4 \sqrt{\rho}} \times\left(\frac{C_{1} \cos \left(\frac{1}{2} \sqrt{4 \mu-\lambda^{2}}\right) \xi-C_{2} \sin \left(\frac{1}{2} \sqrt{4 \mu-\lambda^{2}}\right) \xi}{C_{1} \sin \left(\frac{1}{2} \sqrt{4 \mu-\lambda^{2}}\right) \xi+C_{2} \cos \left(\frac{1}{2} \sqrt{4 \mu-\lambda^{2}}\right) \xi}\right),
$$

and

$u_{2}(x, t)=\left[ \pm \frac{\epsilon \sqrt{4 \mu-\lambda^{2}}}{4 \sqrt{\rho}} \times\left(\frac{C_{1} \cos \left(\frac{1}{2} \sqrt{4 \mu-\lambda^{2}}\right) \xi-C_{2} \sin \left(\frac{1}{2} \sqrt{4 \mu-\lambda^{2}}\right) \xi}{C_{1} \sin \left(\frac{1}{2} \sqrt{4 \mu-\lambda^{2}}\right) \xi+C_{2} \cos \left(\frac{1}{2} \sqrt{4 \mu-\lambda^{2}}\right) \xi}\right)\right] \times e^{i c(x-\sigma t)}$

where $\xi=x+\frac{\varepsilon}{2} t$.

When $\lambda^{2}-4 \mu=0$, we have the rational type

$$
V_{3}(\xi)= \pm \frac{\epsilon C_{2}}{2 \sqrt{\rho}\left(C_{1}+C_{2} \xi\right)}
$$

and

$$
u_{3}(x, t)=\left[ \pm \frac{\epsilon C_{2}}{2 \sqrt{\rho}\left(C_{1}+C_{2} \xi\right)}\right] \times e^{i c(x-\sigma t)}
$$

Case 2: When $\lambda^{2}-4 \mu>0, k_{1}<0$, we have the hyperbolic type

$$
V_{4}(\xi)=\mp \frac{k_{1}}{\sqrt{-k_{1}}} \sqrt{\lambda^{2}-4 \mu} \times\left(\frac{C_{1} \sinh \left(\frac{1}{2} \sqrt{\lambda^{2}-4 \mu}\right) \xi+C_{2} \cosh \left(\frac{1}{2} \sqrt{\lambda^{2}-4 \mu}\right) \xi}{C_{1} \cosh \left(\frac{1}{2} \sqrt{\lambda^{2}-4 \mu}\right) \xi+C_{2} \sinh \left(\frac{1}{2} \sqrt{\lambda^{2}-4 \mu}\right) \xi}\right)
$$

and

$u_{4}(x, t)=\left[\mp \frac{k_{1}}{\sqrt{-k_{1}}} \sqrt{\lambda^{2}-4 \mu} \times\left(\frac{C_{1} \sinh \left(\frac{1}{2} \sqrt{\lambda^{2}-4 \mu}\right) \xi+C_{2} \cosh \left(\frac{1}{2} \sqrt{\lambda^{2}-4 \mu}\right) \xi}{C_{1} \cosh \left(\frac{1}{2} \sqrt{\lambda^{2}-4 \mu}\right) \xi+C_{2} \sinh \left(\frac{1}{2} \sqrt{\lambda^{2}-4 \mu}\right) \xi}\right) \times e^{i \cos (x-\sigma t)}\right.$

where $\xi=x-v t$.

Substituting the solution sets $(3.7,3.8)$ and the corresponding solutions of (2.4) into (3.5), we have the solutions of (3.1) as follows:

Case1: When $\lambda^{2}-4 \mu>0$, we have the hyperbolic type

$$
V_{1}(\xi)= \pm \frac{\epsilon \sqrt{\lambda^{2}-4 \mu}}{4 \sqrt{\rho}} \times\left(\frac{C_{1} \sinh \left(\frac{1}{2} \sqrt{\lambda^{2}-4 \mu}\right) \xi+C_{2} \cosh \left(\frac{1}{2} \sqrt{\lambda^{2}-4 \mu}\right) \xi}{C_{1} \cosh \left(\frac{1}{2} \sqrt{\lambda^{2}-4 \mu}\right) \xi+C_{2} \sinh \left(\frac{1}{2} \sqrt{\lambda^{2}-4 \mu}\right) \xi}\right),
$$

When $k_{1}>0$

$$
V_{5}(\xi)= \pm i \sqrt{k_{1}} \sqrt{\lambda^{2}-4 \mu} \times\left(\frac{C_{1} \sinh \left(\frac{1}{2} \sqrt{\lambda^{2}-4 \mu}\right) \xi+C_{2} \cosh \left(\frac{1}{2} \sqrt{\lambda^{2}-4 \mu}\right) \xi}{C_{1} \cosh \left(\frac{1}{2} \sqrt{\lambda^{2}-4 \mu}\right) \xi+C_{2} \sinh \left(\frac{1}{2} \sqrt{\lambda^{2}-4 \mu}\right) \xi},\right.
$$

$u_{5}(x, t)=\left[ \pm i \sqrt{k_{1}} \sqrt{\lambda^{2}-4 \mu} \times\left(\frac{C_{1} \sinh \left(\frac{1}{2} \sqrt{\lambda^{2}-4 \mu}\right) \xi+C_{2} \cosh \left(\frac{1}{2} \sqrt{\lambda^{2}-4 \mu}\right) \xi}{C_{1} \cosh \left(\frac{1}{2} \sqrt{\lambda^{2}-4 \mu}\right) \xi+C_{2} \sinh \left(\frac{1}{2} \sqrt{\lambda^{2}-4 \mu}\right) \xi}\right] \times e^{i \varepsilon(z-\sigma t)}\right.$ 
When $\lambda^{2}-4 \mu<0, k_{1}<0$; we have the trigonometric type

$V_{6}(\xi)=\mp \frac{k_{1}}{\sqrt{-k_{1}}} \sqrt{4 \mu-\lambda^{2}} \times\left(\frac{C_{1} \cos \left(\frac{1}{2} \sqrt{4 \mu-\lambda^{2}}\right) \xi-C_{2} \sin \left(\frac{1}{2} \sqrt{4 \mu-\lambda^{2}}\right) \xi}{C_{1} \sin \left(\frac{1}{2} \sqrt{4 \mu-\lambda^{2}}\right) \xi+C_{2} \cos \left(\frac{1}{2} \sqrt{4 \mu-\lambda^{2}}\right) \xi}\right)$,

and

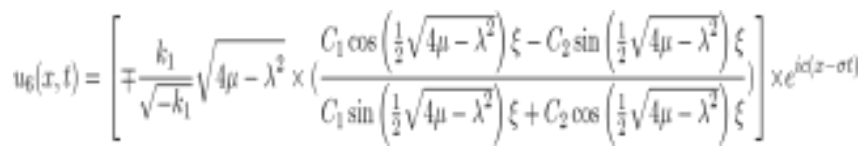

where $\xi=x-v t$.

When $k_{1}>0$

$V_{7}(\xi)= \pm i \sqrt{k_{1}} \sqrt{4 \mu-\lambda^{2}} \times\left(\frac{C_{1} \cos \left(\frac{1}{2} \sqrt{4 \mu-\lambda^{2}}\right) \xi-C_{2} \sin \left(\frac{1}{2} \sqrt{4 \mu-\lambda^{2}}\right) \xi}{C_{1} \sin \left(\frac{1}{2} \sqrt{4 \mu-\lambda^{2}}\right) \xi+C_{2} \cos \left(\frac{1}{2} \sqrt{4 \mu-\lambda^{2}}\right) \xi}\right)$,

and

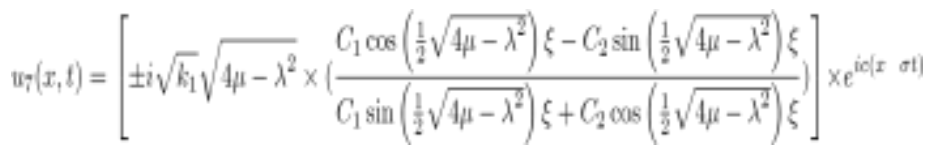

When $\lambda^{2}-4 \mu<0, k_{1}<0$, we have the rational type

$V_{8}(\xi)= \pm \frac{2 k_{1} C_{2}}{\sqrt{-k_{1}}\left(C_{1}+C_{2} \xi\right)}$

and

$u_{8}(x, t)=\left[ \pm \frac{2 k_{1} C_{2}}{\sqrt{-k_{1}}\left(C_{1}+C_{2} \xi\right)}\right] \times e^{i c(x-\sigma t)}$

where $\xi=x-v t$.

When $k_{1}>0$

$$
\begin{gathered}
V_{9}(\xi)= \pm \frac{2 i \sqrt{k_{1}} C_{2}}{\left(C_{1}+C_{2} \xi\right)}, \\
u_{9}(x, t)=\left[ \pm \frac{2 i \sqrt{k_{1}} C_{2}}{\left(C_{1}+C_{2} \xi\right)}\right] \times e^{i c(x-\sigma t)}
\end{gathered}
$$

where $\xi=x-v t$.

\section{Conclusion}

In this paper, the $\left(G^{\prime} / G\right)$-expansion method has been successfully used to obtain travelling wave solutions for the Perturbed Wadati-Segur-Ablowitz equation. The obtained solutions may be important to expose most complex physical phenomena or to find new phenomena.This method has more advantages since it is too difficult to solve complex nonlinear partial differential equations by traditional methods. The procedure is direct and simple that the general solutions of the second order LODE have been well known. The availability of computer systems like Maple or Mathematica facilitates the tedious algebraic calculations. The obtained solutions in this study will be very useful in various physical situations where this equation arise.

\section{References}

[1] Zhang., S., Xia., T., A generalized new auxiliary equation method and its applications to nonlinear partial differential equations, Phys. Lett. A., 363 (2007) 356.

[2] Ablowitz.,M.J., Clarkson, P. A.,Soliton, Nonlinear Evolution Equation and Inverse Scattering, Cambridge University Press, New York, 1991.

[3] R. Hirota, Exact solution of the Korteweg de Vries equation for multiple collisions of solitons, Phys. Rev. Lett. 27 (1971), 1192-1194.

[4] Miurs., M. R. Backlund Transformation, Springer, Berlin, 1978.

[5] Weiss., J., Tabor, G., Carnevale, G., The Weiss Tabor Carnevale (WTC) Painleve test, and its recent perturbative extension, J. Math. Phys. 24 (1983) 522.

[6] Yusufoglu, E., Bekir, A., Solitons and periodic solutions of coupled nonlinear evolution equation by using sine-cosine method, Int. J. Comput. Math., (2006) 83 (12) 915-924..

[7] Wang., M. L., Exact Solution for a Compound KdV-Burgers Equations, Phys. Lett. A., 213 (1996) 279.

[8] M.L.Wang, Y.B.Zhou and Z.B.Li., Application of a homogeneous balance method to exact solutions of nonlinear equations in mathematical physics, Phys. Lett. A, 216, (1996),67-75

[9] Bekir, A., Boz, A., Exact solutions for nonlinear evolution equation using exp-function method, Phys. Lett. A., 372 (2008) 1619-1625. 
[10] Naher, H., Abdullah, F.A.,Akbar, M.A.,New Traveling Wave Solutions of the Higher Dimensional Nonlinear Partial Differential Equation by the Exp-Function Method, Journal of Applied Mathematics, 2012, 14.

[11] H. Naher, F.A. Abdullah, Ahmet Bekir, Some new traveling wave solutions of the modified Benjamin-Bona-Mahony equation via the improved $\left(\mathrm{G}^{\prime} / \mathrm{G}\right)$-expansion method, New Trends in Mathematical Sciences, 3 (1),78-89 (2015)

[12] Kangalgil ,F., Travelling wave solutions of the Schamel-Korteweq-de Vries and Schamel equations, Journal of the Egyptian Mathematical Society, (2016) 24, 526-531.

[13] Naher, H., Abdullah, F. A., The improved (G'/G)expansion method to the $(2+1)$ dimensional breaking soliton equation, Journal of Computational Analysis \& Applications, 16 (2),(2014), 220-235.

[14] Naher,H., and Abdullah,F.A., New generalized and improved $\left(\mathrm{G}^{\prime} / \mathrm{G}\right)$-expansion method for nonlinear evolution equations in mathematical physics, Journal of the Egyptian Mathematical Society, http//dx.doi.org/10.1016/j.joems.11.008.

[15]H. Naher, F. A. Abdullah, The improved (G'/G)expansion method to the $(2+1)$-dimensional breaking soliton equation, Journal of Computational Analysis \& Applications, 16, 2, (2014) 220-235..

[16] Guner, , Bekir, A., Karaca,F., Optical soliton solution of nonlinear evolution equations using ansatz method, Optik (2017) 130 696-701.

[17] Ahmet Bekir, O. Unsal, Exact solutions for a class of nonlinear wave equations by using frst integral method, International Journal of Nonlinear Science, 15 (2),99-110 (2013)

[18]A. El Achab, Ahmet Bekir, Travelling Wave Solutions to the Generalized BenjaminBonaMahony (BBM) Equation Using the First Integral Method, International Journal of Nonlinear Science, 19 (1),40-46 (2015)

[19] Bekir, A., El Achab, A., Traveling Wave Solutions to the $K(m, n)$ equation with generalized evolution Using the First Integral Method , New Trends in Mathematical Sciences, 2 (1),(2014) 12-17.

[20] Inc,M., Kilic,B.,The rst integral method for the perturbed Wadati-Segur-Ablowitz equation with time dependent coefficient, Kuwait J. Sci, 43 (1) (2016) 84-94.
[21] Seyma, T, Bulut H., New exact solutions for generalized Gardner equation, Kuwait J. Sci, 44 (1) (2017) 1-8.

[22] Wazwaz, A.M., The tanh method for travelling wave solutions of nonlinear equations, Math Comput Model, 1543, (2004), 713-723.

[23] Wazwaz, A.M., The tanh method for generalized forms of nonlinear heat conduction and BurgerFisher equations, Applied Mathematics and Computation ,169, (2005), 321-338.

[24]Zhou, Y.B., Wang, M.L., Wang, Y.M., Periodic wave solutions to a coupled KDV equations with variable coefficients, Phys. Lett. A. 308 (2003) 3136.

[25] Kangalgil,F., Ayaz, F.,New exact traveling wave solutions for the Ostrovsky equation, Physics Letters A, (2008) 372 (11):1831-1835.

[26] Kangalgil, F., Ayaz, F., Solitary wave solutions for the $\mathrm{KdV}$ and $\mathrm{mKdV}$ equations by differential transform method. Chaos Soliton Fract. 41(1) (2009) 464472.

[27] He J H, Homotopy perturbation technique Comput Methods Appl. Mech. Eng, 178, (1999), 257-62.

[28] M. L. Wang, X. Z. Li, and J. L. Zhang, The $\left(\mathrm{G}^{\prime} / \mathrm{G}\right)$-expansion method and travelling wave solutions of nonlinear evolution equations in mathematical physics, Physics Letters A, 372, 4,( 2008.), 417-423.

[29]Zhang., J., Wei., X., Lu., Y., A generalized (G'/G)-expansion method and its applications, Phys. Lett. A., 372 (2008) 3653-3658.

[30]Zhang., S., Dong., J.Ba., Sun., Y.,The-expansion method for nonlinear differential-difference equations, Phys. Lett. A., 373 (2009) 905.

[31]Bekir, A., Application of the $\left(\mathrm{G}^{\prime} / \mathrm{G}\right)$-expansion method for nonlinear evolution equations, Phys. Lett. A., 372, (2008), 3400-3406.

[32]Kheiri, H., Moghaddan, MR and Vafaei, V., Application of the $\left(\mathrm{G}^{\prime} / \mathrm{G}\right)$-expansion method for the Burgers, Burgers Huxley and modified Burgers KdV equations, Pramana J. Phys., 7, (2011), 831.

[33] Wang, ML, Li, X and Zhang, J., The (G'/G)expansion method and travelling wave solutions of nonlinear evolution equations in mathematical physics, Phys. Lett. A, 372, (2008), 417.

[34] Alam, M.N., Akbar, M.A., Mohyud-Din, S.T, A novel $\left(G^{\prime} / G\right)$-expansion method and its application 
to the Boussinesq equation, Chinese Physics B , 23, (2014), 2.

[35]H. Naher, F. A. Abdullah, New approach of $\left(G^{\prime} / G\right)$-expansion method and new approach of generalized $\left(\mathrm{G}^{\prime} / \mathrm{G}\right)$-expansion method for nonlinear evolution equation, AIP Advances, 3, (32), (2013) 116.

[36] Bekir, A.,Uygun, F., Exact travelling wave solutions of nonlinear evolution equations by using the $\left(G^{\prime} / G\right)$ - expansion method, Arab Journal of Mathematical Sciences, (2012).18,73-85

[37]Bekir,A., Phys., Application of the (G'/G)expansion method for nonlinear evolution equations, Phys.Lett. A., 372 (2008) 3400-3406.

[38]He,J., A new approach to nonlinear partial differential equations, Communications in Nonlinear Science and Numerical Simulation, 2, 4, (1997), 230-235.

[39] Guner A., Bekir, A., Ünsal, Two reliable methods for solving the time fractional Clannish random Walker s Parabolic equation, Optik 127 (2016) 9571-9577.

[40]Ekici, M., Soliton and other solutions of nonlinear time fractional parabolic equations using extended (G'/G)-expansion method, Optik, 130 ( 2017) 1312-1319.

[41] Guner, O, Atik, A., Kayyrzhanovich, A.A., New exact solution for space-time fractional dierential equations via $\left(\mathrm{G}^{\prime} / \mathrm{G}\right)$-expansion method, Optik, 130 (2017) 696-701.

[42] Wadati., M.,Segur., H., Ablowitz., M.J., A new Hamiltonian amplitude equation governing modulated wave instabilities, J. Phys. Japan. 61, (1992), 1187.

[43] CGR Teh, WK Koo, BS Lee , Jacobian Elliptic Wave Solutions for the Wadati Segur Ablowitz Equation, International Journal of Modern Physics B, 11 (1997) 2849. 